\title{
NEW CONVERSES OF THE JESSEN AND LAH-RIBARIČ INEQUALITIES
}

\author{
ROZARIJA JAKŠIĆ AND JOSIP PEČARIĆ
}

Abstract. New converses of the Jessen and Lah-Ribarič inequalities for continuous convex functions with applications to means, the Hölder inequality, the Hadamard inequality, and the inequalities of Giaccardi and Petrović are given.

Mathematics subject classification (2010): 26A51, 26E60, 97H30.

Keywords and phrases: Positive linear functionals, Jensen's inequality, Lah-Ribarič's inequality, convex functions, generalized means, arithmetic means, Hölder's inequality, Hadamard's inequality, inequalities of Giaccardi and Petrović.

\section{REFERENCES}

[1] P. R. BEESACK, J. E. PEČARIĆ, On the Jessen's inequality for convex functions, J. Math. Anal. 110, 536-552, (1985).

[2] P. S. Bullen, D. S. Mitrinović, P. M. Vasić, Means and their inequalities, D. Reidel Publishing Co., Dordrecht, Boston, Lancaster and Tokyo, (1987).

[3] S. S. DRAGOMIR, Reverses of the Jensen's inequality in terms of first derivative and application, Preprint RGMIA Res. Rep. Coll, 2011 - ajmaa.org

[4] G. H. Hardy, J. E. Littlewood, G. Pólya, Inequalities 1st ed. and 2nd ed., Cambridge University Press, Cambridge, England, (1934, 1952).

[5] B. Jessen, Bemaerkinger om konvekse Funktioner og Uligheder imellem Middelvaerdier I, Mat. Tidsskrift, B, 17-28, (1931).

[6] D. S. Mitrinović, I. B. Lacković, Hermite and convexity, Aequat. Math., 28, 229-232.

[7] J. E. PeČArić, F. Proschan, Y. L. Tong, Convex functions, Partial orderings and statistical applications, Academic Press Inc., San Diego, (1992).

[8] J. E. PeČARIĆ, P. R. BeESACK, On Jessen's inequality for convex functions II., J. Math. Anal. Appl., 118, 125-144, (1986).

[9] M. Petrović, Sur une fonctionnelle, Publ. Math. Univ. Belgrade 1, 149-156, (1932).

[10] P. M. VASIĆ, J. E. PeČARIĆ, On the Jensen inequality for monotone functions I, Anal. Univ. Timişoara 1, 95-104, (1979). 